\title{
ALTERATION TUMOR NECROSIS FACTOR-ALPHA AND MATRIX METALLOPROTEINASE-9 EXPRESSION IN CANDIDA KERATITIS WITH AND WITHOUT STEROID ADMINISTRATION
}

\author{
HAVRIZA VITRESIA ${ }^{1}$, JAMSARI JAMSARI ${ }^{2}$, GONDHOWIHARDJO T ${ }^{3}$, ANDANI EKA PUTRA ${ }^{4}$
}

${ }^{1}$ Department of Ophthalmology, Faculty of Medicine, Andalas University, Padang-Indonesia. ${ }^{2}$ Department of Agriculture, Faculty of Agriculture, Andalas University, Padang-Indonesia. ${ }^{3}$ Department of Ophthalmology, Faculty of Medicine, University of Indonesia, Jakarta-Indonesia. ${ }^{4}$ Department of Microbiology, Faculty of Medicine, Andalas University, Padang-Indonesia. Email: vitresia@gmail.com Received: 08 September 2020, Revised and Accepted: 09 October 2020

\section{ABSTRACT}

Objective: The objective of the study was to determine the severity level of keratitis, inflammatory response, and clinical feature of fungal keratitis associated with tumor necrosis factor-alpha (TNF- $\alpha$ ) and matrix metalloproteinase (MMP)-9 expression, with and without steroid administration.

Methods: The mice corneas were inoculated with $10^{8} \mathrm{CFU}$ Candida albicans (CA), divided into two groups, one group with steroid administration and other group without steroid, and uninoculated cornea is used as the control. The mice eyes were observed for the development of keratitis and assess the clinical score to categorize severity level of the keratitis. After 7 days, they were enucleated for analysis of TNF- $\alpha$ and MMP-9 expression with immunohistochemistry and histopathologic examination.

Results: Results show that the group inoculated with Candida and steroid has more moderate and severe level of keratitis compared to the group inoculated with Candida only. The final clinical score in the group of CA injected with steroid was 5-12 with mean 8.89 (2.67). The histopathological examination confirmed the presence of prominent inflammatory response in steroid group. The mean of TNF- $\alpha$ expression in Candida keratitis with and without steroid injections is 50.1 (8.09) and 31.7 (6.89) consecutively, compared to 16.8 (1.58) of control group. The mean of MMP-9 expression in Candida keratitis with steroid injection is higher than Candida keratitis group without steroid injection and control group, which are 46.6 (4.3), 37.8 (4.96), and 17.2 (1.73), respectively. This difference is statistically significant.

Conclusion: Administration of steroids to Candida keratitis has negative effect to corneal tissue, resulting in more severe inflammatory response associated with increased TNF- $\alpha$ and MMP-9 expressions.

Keywords: Candida keratitis, Tumor necrosis factor-alpha expression, Severity.

(c) 2020 The Authors. Published by Innovare Academic Sciences Pvt Ltd. This is an open access article under the CC BY license (http://creativecommons. org/licenses/by/4. 0/) DOI: http://dx.doi.org/10.22159/ajpcr.2020.v13i11.39675

\section{INTRODUCTION}

Fungal keratitis is a severe ocular infection and can cause corneal opacity, blindness, even loss of eyeballs, and its challenging disease to diagnose and treat. In India and Southeast Asia, fungal keratitis is still a particular problem, where the prevalence is increasing in hot climates [1-3]. Candida has been documented as a frequent cause of fungal keratitis in temperate climates, it is identified as the cause of $52 \%$ fungal keratitis. It almost exclusively occurs in patients with ocular or systemic disease and is considered relatively uncommon among healthy patient [4].

The risk factors for fungal keratitis are ocular trauma with vegetative matter, abuse of topical steroid, and contact lens wearer. Topical steroid use is an important risk factor aside from ocular trauma, due to the extensive use of this drug in ocular surface disease (OSD) cases. Nielsen study (2015) shows that prior topical steroid treatment was commonly found in $44 \%$ of fungal keratitis cases [5]. Use of this drug in early stage of OSD is said to create the impression of reduced inflammation, while producing immunosuppressive condition in ocular surface. In fungal keratitis, inappropriate use of topical steroid may be related to the inflammation masking effect of previous steroid topical used in early stage infection. This finding may also make early diagnosis of fungal keratitis difficult. On the other hand, steroid use can promote fungal proliferation and worsen infection due to severe inflammatory side effects and they also affect the delay of epithelial regeneration [6]

The previous studies have shown that the immune system plays an important role in the pathogenesis of fungal keratitis. Corneal epithelium as a corneal barrier in fighting pathogen infections plays an important role in innate immunity. Activation of macrophages as presenting cell antigens that play a core role in innate immunity against fungal infections also increases the production of cytokines, chemokines, and polymorphonuclear (PMN) infiltration. However, if the immune response occurs excessively, it can lead to more severe corneal infections [7,8]. Tumor necrosis factor-alpha (TNF- $\alpha$ ) is a cytokine in systemic inflammation and members of the cytokine group that stimulates acute phase reactions mainly produced by macrophages or other cells. TNF- $\alpha$ is known to have a role in the immune response to the invasion of bacterize, fungi, viruses, and certain parasites. There is an induction of the production of several cytokines, one of which is TNF- $\alpha$, which will only appear if there has been a defect in the corneal epithelium in Candida albicans (CA) infection. TNF- $\alpha$ has the effect and potentiation of PMN activity. PMN, along with macrophages, has the role of clearing fungal invasion through phagocytosis $[9,10]$.

Moreover, increased expression of TNF- $\alpha$ will trigger the increased of proteolytic enzymes activity and expression. One of the important proteolytic enzymes in Candida keratitis is matrix metalloproteinase (MMP)-9. MMP-9 contributes significantly to the healing process of Candida keratitis, but an increased expression of this proteolytic enzyme could cause severe inflammatory process, heavier tissue damage, corneal melt, and even perforation $[11,12]$. The aim of this study was to analyze steroid effect on Candida keratitis based on the severity level of keratitis, inflammatory response of the tissue, expression of TNF- $\alpha$, and MMP-9 relating to inflammation response of corneal tissue in Candida keratitis.

\section{METHODS}

This study was performed in experimental research. The mice corneas were divided into three groups, uninoculated eyes as a control (A) and 
two other groups were inoculated with CA (B), and groups inoculated with $\mathrm{CA}$ with additional steroid administration $(\mathrm{C})$.

CA obtained from the Laboratory of Microbiology, Faculty of Medicine, UNAND, was first rejuvenated. Yeast rejuvenated is done by inoculating 1 ose of pure of yeast culture into Sabouraud medium, then incubated at $37^{\circ} \mathrm{C}$ for $72 \mathrm{~h}$ under aerobics conditions. CA is diluted by mixing 1 ose of yeast into a test tube containing $2 \mathrm{ml}$ sterile saline. Then, homogenous with vortex and turbidity standardized on a scale of 0.5 McFarland (1.5 $\times 10^{8} \mathrm{CFU} / \mathrm{ml}$ ).

\section{Population}

Mice included in the study were of Animal House of the Medical Faculty, Andalas University (UNAND), Padang, Indonesia. Mice that fulfill inclusion criteria were chosen as sample for this study. The sample size (n) was calculated by Federer formula. Inclusion criteria of the study were Wistar mice, body weight between 150 and 250 grams, good health, and without other eye disorders. The exclusion criteria of the study were mice that experienced any disease during the study.

Before inoculation, mice were put under with ketamine $(37.5 \mathrm{mg} / \mathrm{mL})$, xylazine $(1.9 \mathrm{mg} / \mathrm{mL})$, and acepromazine $(0.37 \mathrm{mg} / \mathrm{mL})$ anesthetics. Cornea of the right eye of mice was injured using knife No. 11 to make a defect forming a grid pattern and received a $5 \mu \mathrm{l} \mathrm{CA}\left(1 \times 10^{8} \mathrm{CFU}\right)$ inoculum which was then applied to injured cornea. The eyelids were rubbed for a few seconds to distribute inoculum fully on the surface of the cornea. Steroids were given topically and injected, prednisolone eye drops and methyl prednisolone injection at $100 \mathrm{mg} / \mathrm{kg}$ body weight. An intramuscular injection was administered 3 days before, 1 day before, and 1 day after corneal inoculation with CA. Uninoculated mice were used as control comparisons group in the study.

Mice were observed and scored every day to categorize the severity of keratitis. Visual scoring system used in this study was developed by Wu et al. Grades 0-4 are determined for each criterion; the area of corneal opacity, corneal opacity density, and surface irregularity. Total score ranging $0-12$. A total score of 5 or less were categorized as mild disease, 6-9 categorized as moderate, and score of more than 9 was severe. Visual scoring for Candida keratitis is shown in Table 1.

\section{IHC examination}

Mice were killed, and the eyes were enucleated 7 days after inoculation and then processed for histopathological examination and IHC examination for TNF- $\alpha$ and MMP-9 expression measurement. During this research, if dead mice are found, the eyeball enucleation would still be carried out and included as sample. The sample for histopathological examination was formalin fixed and paraffin-embedded procedures. The sections were deparaffinized with hematoxylin and eosin and periodic acid-Schiff staining. The examination was performed in Anatomical Pathology Laboratory, Faculty of Medicine, Andalas University, Padang, Indonesia. Expression of TNF- $\alpha$ and MMP-9 measured with immunohistochemistry and quantitative value was analyzed with isolate the brown area in immunoperoxidase stain and measured using ImageJ program. An analysis of TNF- $\alpha$ and MMP-9 expression was performed in each group.

Preparation of this research was done carefully to ensure the mice experience a good environment. Food and drink were given as needed. Thus, it can be ascertained that the sample gets the same treatment.
Mice are maintained properly according to existing requirements to obtain conditions and requirements suitable for research. Mice that were randomly selected are put into a trial cage that had been prepared for 1 week to make adjustments as a stable environment before conducting research.

\section{Statistic of analysis}

Data were analyzed after passing normality and homogeneity tests. The data normality test used was Kolmogorov-Smirnov method, while the homogeneity of the data was tested by Levene test. Data analysis was followed by t-test and one-way ANOVA to compare the mean of all treatment groups and control groups (without treatment). If the oneway ANOVA test results in the conclusion that Ho is rejected, it means that there is a significant difference and the analysis is continued with a multiple comparison test, in this case, the least significant difference is used at $5 \%$ level.

\section{Research ethics}

This study has received ethical approval from the Research Ethics Commission of the Faculty of Medicine, Andalas University.

\section{RESULTS}

After the inoculation with $10^{8} \mathrm{CFU}$ of $\mathrm{CA}$, all eyes of mice were visually observed daily for keratitis for 7 days. The severity of keratitis was scored visually. Eyes inoculated with CA without steroid injection developed keratitis with final clinical score of 3-7 with mean 4.33 (1.65), meanwhile, eyes with CA inoculated and steroid have final clinical score of 5-12 with mean 8.89 (2.67).

This final clinical score presented the severity level of keratitis, and the results showed that eyes inoculated with CA and steroid administrations had more moderate and severe keratitis than without steroid injection. This group had more extensive area of corneal opacity, swelling, and perforation (Table 2).

After enucleated, the corneal tissues were examined for histopathology. HE examination showed the inflammation with neutrophilic infiltration and stromal destruction was prominent in eyes inoculated with CA with steroid and less severe in eyes inoculated with CA only. Periodic acid-Schiff (PAS) staining showed the microorganism with PAS positive, but most of the organism not intact. The results are shown in Figs. 1 and 2.

Quantitative measurement of TNF- $\alpha$ and MMP-9 is done by ImageJ immunoperoxidase program. Results show that Candida keratitis with steroid group has higher TNF- $\alpha$. In Candida keratitis group injected with steroid, TNF- $\alpha$ expression is higher than keratitis group without steroid injection and control group.

This increase in TNF- $\alpha$ expression is followed by increase in MMP-9 expression, and both expressions are higher in steroid-injected keratitis group compared to the other two groups. This difference is statistically significant $(\mathrm{p}<0.05)$ (Table 3).

Excessive MMP-9 expression causes profuse extracellular matrix degradation, associated with tissue damage and keratitis severity level. This study also compares MMP-9 expression with keratitis severity level (mild, moderate, and severe). Results show that MMP-9 expression rises 2.7 times higher in Candida keratitis with steroid group, in comparison

Table 1: Visual scoring for Candida keratitis

\begin{tabular}{|c|c|c|c|c|}
\hline Criteria & Grade 1 & Grade 2 & Grade 3 & Grade 4 \\
\hline Area of corneal opacity & $1-25 \%$ & $26-50 \%$ & $51-75 \%$ & $75-100 \%$ \\
\hline Density of corneal opacity & $\begin{array}{l}\text { Slight cloudiness, outline of iris } \\
\text { and pupil discernable }\end{array}$ & $\begin{array}{l}\text { Cloudy, but outline of iris and } \\
\text { pupil remain visible }\end{array}$ & Cloudy, opacity not uniform & Uniform opacity \\
\hline Surface regularity & Slight surface irregularity & Rough surface, some swelling & $\begin{array}{l}\text { Significant swelling, crater or } \\
\text { serious descemetocele formation }\end{array}$ & $\begin{array}{l}\text { Perforation or } \\
\text { descemetocele }\end{array}$ \\
\hline
\end{tabular}


to normal group. This raise shows that an increase in MMP-9 expression correlates with keratitis severity level.

Statistical analysis toward MMP-9 expression and keratitis severity level was done by one-way ANOVA method. Post hoc and Bonferroni test were done to identify each group that shows any difference. There was a significant difference in MMP-9 expression between normal and keratitis group according to keratitis severity level $(p<0.05)$. There was an increase in MMP-9 in accordance with keratitis severity level, where the highest MMP-9 level was obtained in severe keratitis group, but this difference is not statistically significant $(\mathrm{p}>0.05)$. This result is shown in Fig. 3.

\section{DISCUSSION}

Infection with Candida has been documented as a frequent cause of fungal keratitis in temperate climates. It almost exclusively occurs in patients with ocular or systemic disease and is considered relatively uncommon among healthy patients [4]. Fungal keratitis in the previous

Table 2: Severity level of Candida keratitis

\begin{tabular}{llll}
\hline Groups & Mild (\%) & Moderate (\%) & Severe \\
\hline B (CA inoculated) & $6(66.7)$ & $3(33.3)$ & - \\
C (CA inoculated + steroid) & $1(11.1)$ & $4(44.45)$ & $4(44.45)$ \\
\hline
\end{tabular}

Table 3: Comparison of TNF- $\alpha$ and MMP-9 expression in each group

\begin{tabular}{lllll}
\hline Groups & TNF- $\boldsymbol{\alpha}$ expression & $\mathbf{p}$ & MMP-9 expression & $\mathbf{p}$ \\
\hline A (control) & $16.7 \pm 1.58$ & 0.00 & $17.2 \pm 1.73$ & 0.00 \\
B (CA) & $31.7 \pm 6.89$ & & $37.8 \pm 4.95$ & \\
C (CA + & $50.1 \pm 8.09$ & & $46.5 \pm 4.30$ & \\
steroid) & & & & \\
\hline
\end{tabular}

MMP: Matrix metalloproteinase, TNF: Tumor necrosis factor

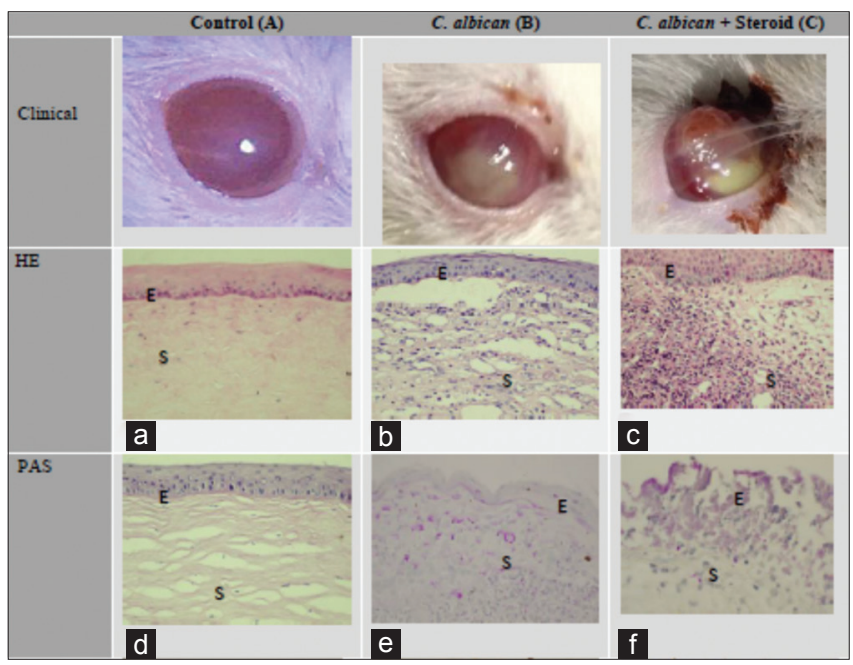

Fig. 1: Comparison of clinical feature, histopathology (HE and Periodic acid-Schiff staining), and immunohistochemistry (IHC) examination. Uninoculated mice as a control (left), inoculated mice with Candida albicans (middle), and inoculated mice with additional steroid administration (right). The upper row shows clinical manifestations in three groups, steroid-injected keratitis group (C) shows worse keratitis severity level. HE staining shows difference in inflammatory responses. The difference can be observed by leukocytes infiltration in corneal stroma (S) and surface epithelial degeneration (E) in the three groups, where the Candida keratitis group with steroid injection generates greater inflammatory response studies was shown to be more likely to perforate the cornea and leads to irreversible changes [1]. In addition, Srinivas study shows that with therapeutic keratoplasty treatment in severe fungal keratitis, graft rejection is seen in all the cases with poor visual outcome but with maintenance of structural integrity [13].

One of the risk factors for fungal keratitis is inappropriate use of topical steroid, and the previous study showed that history of topical steroid administration was found in $44 \%$ of fungal keratitis [5]. It has been recognized that tissue destruction in microbial keratitis results from destructive enzymes released by the host and toxins from microbes. Corticosteroid effect in reducing the host inflammatory response triggered by various enzymes and toxins responsible for corneal destruction could be considered as an excuse to justify its use. Several studies have demonstrated that using topical corticosteroid as adjuvant therapy in bacterial keratitis may reduce the visual impairment by reducing the scar size $[14,15]$. The result of clinical trial, The Steroids for Corneal Ulcers Trial points in favor of using topical corticosteroid in bacterial keratitis. They did not find the differences in corneal perforation as a serious adverse event, while Sarchahi showed that the use of dexamethasone $0.1 \%$ associated with acetylcysteine could increase mean healing time, but its administration should be delayed until the completion of the epithelial defect $[16,17]$

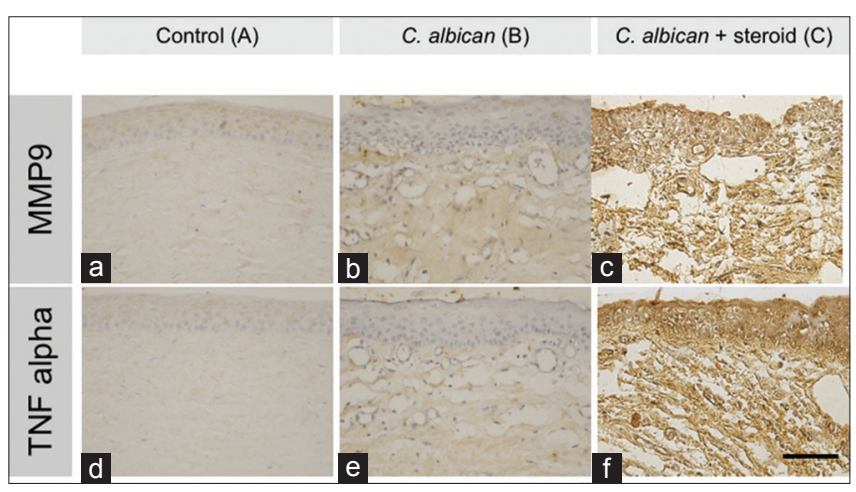

Fig. 2: Tumor necrosis factor-alpha (TNF- $\alpha$ ) and matrix metalloproteinase-9 (MMP-9) expression in immunoperoxidase assay with TNF- $\alpha$ and MMP-9 antibodies in the three groups. The difference in brown stain among the group shows increase in TNF- $\alpha$ and MMP-9 expression. Candida keratitis group inoculated with steroid (Fig. $c$ and $f$ ) has higher TNF- $\alpha$ and MMP-9 expression than Candida keratitis group without steroid and control group. The quantitative value of TNF- $\alpha$ expression level was analyzed with isolate the brown area in immunoperoxidase stain and measured using ImageJ program (ImageJ $1.49 \mathrm{v}$ software, National Institute of Health, Bethesda, MD, USA). (Scale: $200 \mu \mathrm{m}$ )

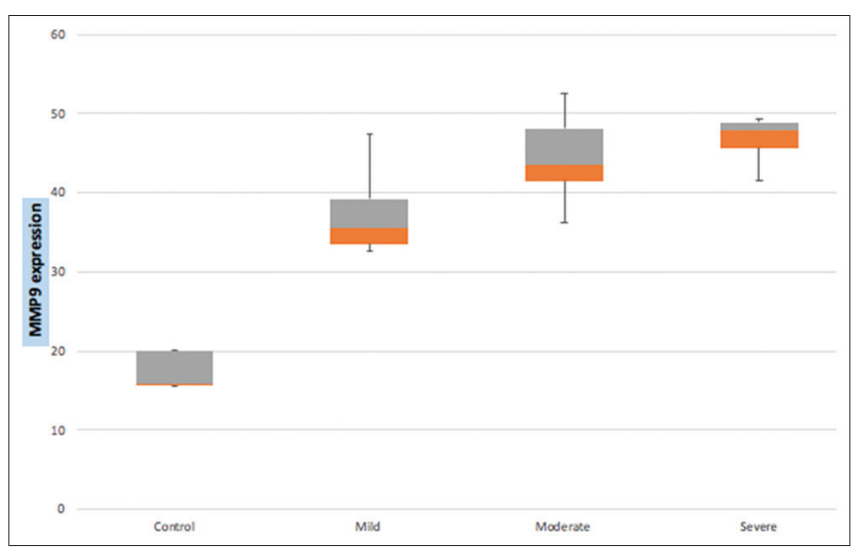

Fig. 3: Matrix metalloproteinase-9 expression according severity level of keratitis 
Topical corticosteroid drops are known to inhibit neutrophil chemotaxis and are, thus, believed to reduce the collagenase and cytokine burden that leads first to ulceration and then to scarring [17]. Even though steroid use has not been said to produce negative effect in bacterial keratitis, the question regarding its use in fungal keratitis remains unanswered. Steroid is said to create the impression of reduced inflammation and produce immunosuppressive condition in ocular surface. Steroid can promote fungal proliferation and worsen infection due to severe inflammatory side effects and they also cause delay of epithelial regeneration $[16,18]$.

This study found that the Candida keratitis group with steroid injection had worse severity level compared to the group without. Severe level keratitis was found only in steroid-administered Candida keratitis group. This can be observed by higher clinical score in steroid-administered Candida keratitis, compared to the other groups. HE examination supports this clinical finding and showed that inflammation with neutrophilic infiltration and stromal destruction was prominent in eyes inoculated with CA and steroid and less severe in eyes only inoculated with CA. Meanwhile, PAS staining showed the microorganism with PAS positive, but most of the organism was not intact. Histopathology examination found that tissue destruction was more related to inflammatory response than the pathogen itself.

After corneal epithelial defect occur and damaged the basal membrane, TNF- $\alpha$ and other cytokines will be expressed to the corneal stroma and play a role in promoting apoptosis, leukocyte recruitment, promoting epithelial migration, upregulation of growth factors, and mediating the stromal healing response. TNF- $\alpha$ is an important factor in the relationship of specific immunity and triggered an inflammatory reaction. Its expression was amplified in OSD with heavy inflammatory response followed by PMN recruitment and progression of the condition $[7,10]$. This study analyzed experimental fungal keratitis with or without administration of systemic and topical steroid, compared to normal cornea. TNF- $\alpha$ expression was elevated in fungal keratitis, and in fungal keratitis with steroid administration, this expression was heightened up to 3 times the normal amount. This is in accordance with the clinical manifestation in mice corneal tissue, where the fungal keratitis group with steroid administration, more than half had moderate-to-severe severity level. Meanwhile, fungal keratitis group without steroid administration had no case of severe keratitis. This finding is supported by histopathological examination that demonstrated differences in inflammatory responses among the three groups. It can also be observed that tissue damage that occurs in fungal keratitis was not only caused by fungal pathogens but also by excessive inflammatory response.

TNF- $\alpha$ plays an important role in corneal epithelial diseases associated with ocular inflammation. TNF- $\alpha$, a pro-inflammatory cytokine, contributes to ocular inflammation such as that associated with infection, injury, and dry eye, and its concentration in tear fluid has been markedly increased in patient with OSD. Ocular inflammation associated with OSDs has been shown to affect corneal structure and function [19].

TNF-a induces the expression of MMPs in certain cell types. Activation of MMP-9 at the ocular surface in experimental dry eye has been shown to promote disruption of corneal epithelial barrier function as a result of the loss of tight junctions from superficial corneal epithelial cells [19]. This study also indicated that TNF- $\alpha$ increase was followed by a rise in MMP-9 expression. This increase in MMP-9 happened in accordance with disease progressivity, and the highest amount of MMP-9 was found in severe fungal keratitis. Corneal injury also stimulates the increase of MMP-9 and the result of some studies supports the hypothesis that MMP-9 plays an important role in keratomycosis progression. Yuan et al. in their study confirmed that MMP-8 and -9 have increased expression in fungal keratitis and suggest that these MMPs signal leukocyte extravasation and chemotaxis [20]. MMPs can also be released by corneal epithelial cells and keratocyte to potentiate the degradation of corneal epithelial basement membrane and stroma that occurs with fungal adherence and invasion. MMP-9 increases immediately after superficial corneal wounding and fungal infection perpetuates its upregulation $[11,20]$. Kaufman also said that MMP-9 is produced in OSD and is important because it is a reliable indicator of the presence of some of ocular inflammation. The presence of elevated levels of MMP-9 indicates an active ocular surface inflammation as part OSD [21].

Moreover, increased expression of TNF- $\alpha$ will trigger proteolytic enzymes activity and expression. MMP-9 contributes significantly to the healing process of Candida keratitis, but an inflated expression of this proteolytic enzyme could cause severe inflammatory process, heavier tissue damage, corneal melt, and even perforation. Recently, studies pointed in favor of collagenase inhibitor administration as treatment modality in infectious keratitis.

This study shows that steroids administration to fungal keratitis can intensify infection due to a severe inflammatory response. Excessive inflammatory response will damage the corneal tissue, aggravate the disease progression, and increase the severity level of keratitis. Fungal keratitis with previous steroid administration was also accompanied by an increase in TNF- $\alpha$ expression, a pro-inflammatory cytokine that contributes to ocular inflammation. TNF- $\alpha$ induces the expression of MMPs, and then, the increase of TNF- $\alpha$ expression was followed by a rise in MMP-9 expression. The increase of TNF- $\alpha$ and MMP-9 expressions happened in accordance with disease progressivity, and the highest amount was found in severe fungal keratitis. Hence, it should be emphasized that the use of steroids is contraindicated if fungal infection is suspected and clinicians also should be cautious when prescribing steroids for suspected cases of infectious keratitis.

\section{CONCLUSION}

Administration of steroids to Candida keratitis has negative effect to corneal tissue, resulting in more severe inflammatory response, and increase the severity level of keratitis. This corneal disease progression was accompanied by an increase in TNF- $\alpha$ expression and then followed by a rise in MMP- 9 expression. The highest of TNF- $\alpha$ and MMP9 expressions happened in accordance with disease progressivity and was found in severe fungal keratitis.

\section{ACKNOWLEDGMENT}

The authors would like to thank Medical Faculty Andalas University for providing the facilities to conduct the research.

\section{AUTHORS' CONTRIBUTIONS}

Havriza Vitresia concepted the research, provided the methods, collected the data, and authored the manuscript. Jamsari and T. Gondhowihardjo managed the literature searches and interpreted the data. Andani Eka Putra performed the statistical analysis.

\section{AUTHORS' FUNDING}

No any source of support for the authors to disclose and all the works are supported by the authors.

\section{CONFLICTS OF INTEREST}

All the authors declare that they have no conflicts of interest in publishing this research article.

\section{REFERENCES}

1. Ansari Z, Miller D, Galor A. Current thoughts in fungal keratitis: Diagnosis and treatment. Curr Fungal Infect Rep 2013;7:209-18.

2. Achyarya Y, Achyarya B, Karki P. Fungal keratitis: Study of increasing trend and common determinants. Nepal J Epidemiol 2017;7:685-93.

3. Lalitha P, Prajna NV, Kabra A. Risk factors for treatment outcome in fungal keratitis. Ophthalmology 2006;113:526-30.

4. Kenia VP, Kenia RV, Pirdankar OH. Diagnosis and management protocol of acute corneal ulcer. Int J Health Sci Res 2020;10:69-78.

5. Nielsen SE, Nielsen E, Julian HO, Lindegaard J, Hojgaard K, Ivarsen A, et al. Incidence and clinical characteristic of fungal keratitis in a Danish 
population from 2000-2013. Acta Ophthalmol 2015;93:54-8.

6. Cho $\mathrm{CH}$, Lee SB. Clinical analysis of microbiologically proven fungal keratitis according to prior topical steroid use: A retrospective study in South Korea. Br Med J 2019;19:207.

7. Qin Y, Zhang L, Xu Z, Zhang J, Jiang Y, Cao Y, et al. Innate immune cell response upon Candida albicans infection. Virulence 2016;7:512-26.

8. Stern ME, Schaumburg CS, Dana R, Calonge M, Niederkorn JY, Pflufelder SC. Autoimmunity at the ocular surface: Pathogenesis and regulation. Mucosal Immunol 2010;3:425-42.

9. Hassell JR, Birk DE. The molecular basis of corneal transparency. Exp Eye Res 2010;91:326-35.

10. Zhang Y, Liang Q, Liu Y, Pan Z, Baudouin C, Labbe A, et al. Expression of cytokine in aqueous humor from fungal keratitis patients. BMC Ophthalmol 2018; 18:105.

11. Mitchel BM, Wu TG, Chong E, Pate JC, Wilhelmus KR. Expression of matrix metalloproteinase 2 and 9 in experimental corneal injury and fungal keratitis. Cornea 2007;26:589-93.

12. Dong X, Shi W, Zeng Q, Xie L. Roles of adherence and matrix metalloproteinase in growth patterns of fungal pathogens in cornea. Curr Eye Res 2005;30:613-20.

13. Srinivas D. Therapeutic keratoplasty in fungal keratitis. J Evid Based Med Healthc 2014;1:1047-60.
14. Shinivasan M. Corticosteroids: A possible treatment for corneal ulcers? Expert Rev Opthalmol 2012;7:1-3.

15. Palliora S, Henry CR, Amescua G, Alfonso EC. Role of steroid in the treatment of bacterial keratitis. Clin Ophthalmol 2016;10:179-86.

16. Srinivasan M, Mascarenhas J, Rajaraman R. Corticosteroid for bacterial keratitis. The steroid for corneal ulcers trial (SCUT). Arch Ophthalmol 2012:130:143-50.

17. Sarchahi AA, Parizi AM, Eghtedan M. Effect of different treatment regimen with dexamethasone and acetylcysteine on corneal wound healing in rabbits. Iran J Med Sci 2011;36:188-95.

18. Austin A, Lietman T. Update on the management of infectious keratitis. Ophthalmology 2017;124:1678-89.

19. Kimura K, Morita Y, Orita T, Haruta J, Takeji Y, Sonoda KH. Protection of human corneal epithelial cells from TNF- $\alpha$-induced disruption of barrier function by rebamipide. Invest Ophthalmol Vis Sci 2013;54:2752-60.

20. Yuan X, Mitchell BM, Wilhelmus KR. Expression of matrix metalloproteinases during experimental Candida albicans keratitis. Invest Ophthalmol Vis Sci 2009;50:737-42.

21. Kaufman HE. The practical detection of MMP-9 diagnoses ocular surface disease and may help prevent its complications. Cornea 2013;32:211-6. 